\title{
Understanding photosynthesis in a spatial-temporal multiscale: The need for a systemic view
}

\author{
Milton C. Lima Neto (1) - Fabricio E. L. Carvalho (1) - Gustavo M. Souza • \\ Joaquim A. G. Silveira
}

Received: 20 January 2021 / Accepted: 26 March 2021 / Published online: 4 April 2021

(C) Brazilian Society of Plant Physiology 2021

\begin{abstract}
In October 2020, at the peak of the COVID-19 pandemic, a group of young Brazilian photosynthesis researchers organized the 1st Brazilian Symposium on Photosynthesis. The event was free and online, with the presence of important guest speakers from all over the world, who discussed their recent works on topics related to the future and perspectives of photosynthesis research. Summarizing the expectations of this symposium we highlighted the importance of adopting a systemic perspective for a
\end{abstract}

M. C. L. Neto

Biosciences Institute, State University of São Paulo UNESP, Coastal Campus, São Vicente,

SP, Brazil

F. E. L. Carvalho · J. A. G. Silveira ( $\square)$

LABPLANT, Department of Biochemistry and Molecular Biology, Federal University of Ceará, Av. Humberto

Monte SN, Campus do Pici, B1. 907, Fortaleza,

CE CEP 60451-970, Brazil

e-mail: silveira@ufc.br

F. E. L. Carvalho

e-mail: fcarvalho@agrosavia.co

F. E. L. Carvalho

Colombiana de Investigación Agropecuaria - Agrosavia. Centro de Investigación La Suiza - Rionegro, Santander, Colombia

\section{G. M. Souza}

Laboratory of Plant Cognition and Electrophysiology

(LACEV), Department of Botany, Institute of Biology,

Federal University of Pelotas, Pelotas,

RS, Brazil better understanding of photosynthesis as a complex and dynamic process. Plants are modular and selfregulating presenting metabolic redundancy and functional degeneration. Among the various biological processes, photosynthesis plays a crucial role in promoting the direct conversion of light energy into carbon skeletons for support growth and productivity. In the past decades, significant advances have been made in photosynthesis at the biophysical, biochemical, and molecular levels. However, this myriad of knowledge has been insufficient to answer crucial questions, such as: how can we understand and eventually increase photosynthetic efficiency and yield in crops subjected to adverse environment related to climate-changing? We believe that a crucial limitation to the whole comprehension of photosynthesis is associated with a vastly widespread classic reductionist view. Moreover, this perspective is commonly accompanied by non-integrative, simplistic, and descriptive approaches to investigate a complex and dynamic process as photosynthesis. Herein, we propose the use of new approaches, mostly based on the Systems Theory, which certainly comes closer to the real world, such as the complex systems that the plants represent.

Keywords Integrative approaches · Network' . Photosynthesis · Systemic Theory 


\section{Introduction: contributions of the 1st Brazilian symposium on photosynthesis to face new challenging scenarios}

To foster and support discussions regarding photosynthesis prospects in a climate change scenario and aiming to put Brazil in a vanguard position in the world of plant science, the 1st Brazilian Symposium on Photosynthesis (IBSP) was held with the theme "Perspectives to improve photosynthetic efficiency in a climate change scenario". In this online event, we discussed the biological and social problems of food security and photosynthetic efficiency under climate change. Despite the SARS-CoV-2 pandemic's challenging times, we dare to create this symposium, which has long been a gap in Brazil and Latin America. The IBSP was attended by more than 15 internationally renowned researchers that were kind enough to share their time, recent finds and perspectives on photosynthesis.

Calvin-Benson-Bassham cycle (CBB) reactions, photosynthetic electron transport, the trade-off of photosynthesis and stress tolerance, carbohydrate and ROS signalling, $\mathrm{N}$ metabolism, photorespiration, elevated $\mathrm{CO}_{2}$, thermal acclimation, C3-CAM modes, stomata regulation, and the importance of cell wall mesophyll conductance and photosynthesis were some of the vanguard topics discussed in this event, with more than a thousand participants that had the opportunity to present their research by oral presentations and abstracts. The symposium, supported by the Brazilian Society of Plant Physiology, promoted the consolidation of networks and collaborations among different research groups in Brazil and worldwide. It was clearly shown that although we are still a developing country, currently experiencing severe problems of funding and promotion for science, we can carry out high-quality international research. The enthusiasm of young researchers, strengthened by the seniors' experience, shows us an arduous but promising path for studying photosynthesis in Brazil for the next generation.

In this context, with the main objective of supporting further discussions and adding new insights to face challenging and complex scenarios, we intend to stimulate a critical perspective on the current studies on photosynthesis with this opinion paper, strengthening towards a systemic view for this field.

\section{The need for integrative approaches to discover a new world of photosynthesis}

Plants are often considered as modular organisms, in which the parts are semi-autonomous or even totally independent each other. In this vein, plants would be nothing more than the "sum of their parts", lacking any level of integrative organization. That is a philosophical concept of plants grounded in a strong materialist and reductionist view. However, it is not the only way to build a consistent and robust science of plants. A complementary counterbalance is given by the perspective of plants as self-organized systems, which their modular parts are integrated by coherent complex signalling systems allowing the emergence of collective behaviour instead only autonomous local responses. In other words, paraphrase Aristotle, "plants are totalities exceeding the sum of their parts" (Souza et al. 2016a; Lüttge, 2021). Therefore, due to such characteristics, a whole understanding of the functioning of these organisms incurs a very complex task.

Although this great complexity has been accepted by most plant scientists, as far as we can see, many published studies still adopt a fragmented, reductionist, and simplistic models, which impair a satisfactory global and integrated understanding of most phenomena related to plant functioning. Nevertheless, in the last decades, extraordinary advances in understanding some plant functions, remarkably photosynthesis, were achieved, under the perspective of mechanistic and reductionist thinking reached by simplified models employing mainly artificial controlled conditions. Indeed, associated with the utilization of powerful techniques involving genetics, biophysical, biochemical, molecular and multi-omics, plant science have generated a large volume of data and significant progress (Silveira and Carvalho 2016; Li et al. 2019; Sousa et al. 2019; Cunha et al. 2019). Yet, the integration of this vast amount of data, and above all, the translation of this large volume of information to concrete advances related to the understanding of photosynthesis, as well as plant physiology as a whole, for the "real world" conditions, is still incomplete (Amaral and Souza 2017).

Oxygenic photosynthesis converts sunlight into chemical energy supporting the survival of virtually all higher life forms (Nelson and Ben-Shem 2004). Although photosynthesis is well described in terms of 
its biochemical and biophysical machinery, showing the analytical steps of light harvest, oxygen evolution, electron transport, and $\mathrm{CO}_{2}$ assimilation by the Calvin-Benson-Bassham cycle, it is, conversely, a complex, highly dynamic, and spatio-temporal scales up dynamic event (Fig. 1). The time courses that occur throughout photosynthetic reactions range from femtoseconds to days, highlighting the high complexity of this process. Accordingly, the apparatus involved in all photosynthetic activities scale up from single protein complexes bonded to the thylakoid lamella in chloroplasts to the whole ecosystem, building up entire forests from fixing carbon dioxide diffused in atmosphere powered by sunlight. Thus, the photosynthetic study is a prime example of research that requires a combined effort among numerous disciplines, including quantum mechanics, biophysics, biochemistry, molecular and structural biology, physiology, ecology among others (Wegner and Lüttge 2019).

In other words, understanding photosynthesis is to understand which scaling up rules are taking place. Furthermore, this is a huge problem, especially when we need to consider that all processes, regardless of a specific scale, are embedded in a "noisy" environment! For instance, Bertolli and Souza (2013) showed that the photosynthetic responses of soybean plants, subjected to a same water deficit stimulus, were significantly affected by specific environmental fluctuations of abiotic factors (temperature, light and air humidity). However, their averages were pretty much similar between independent experiments. Moreover, each hierarchical level of plant organization (from photochemical efficiency up to biomass production)

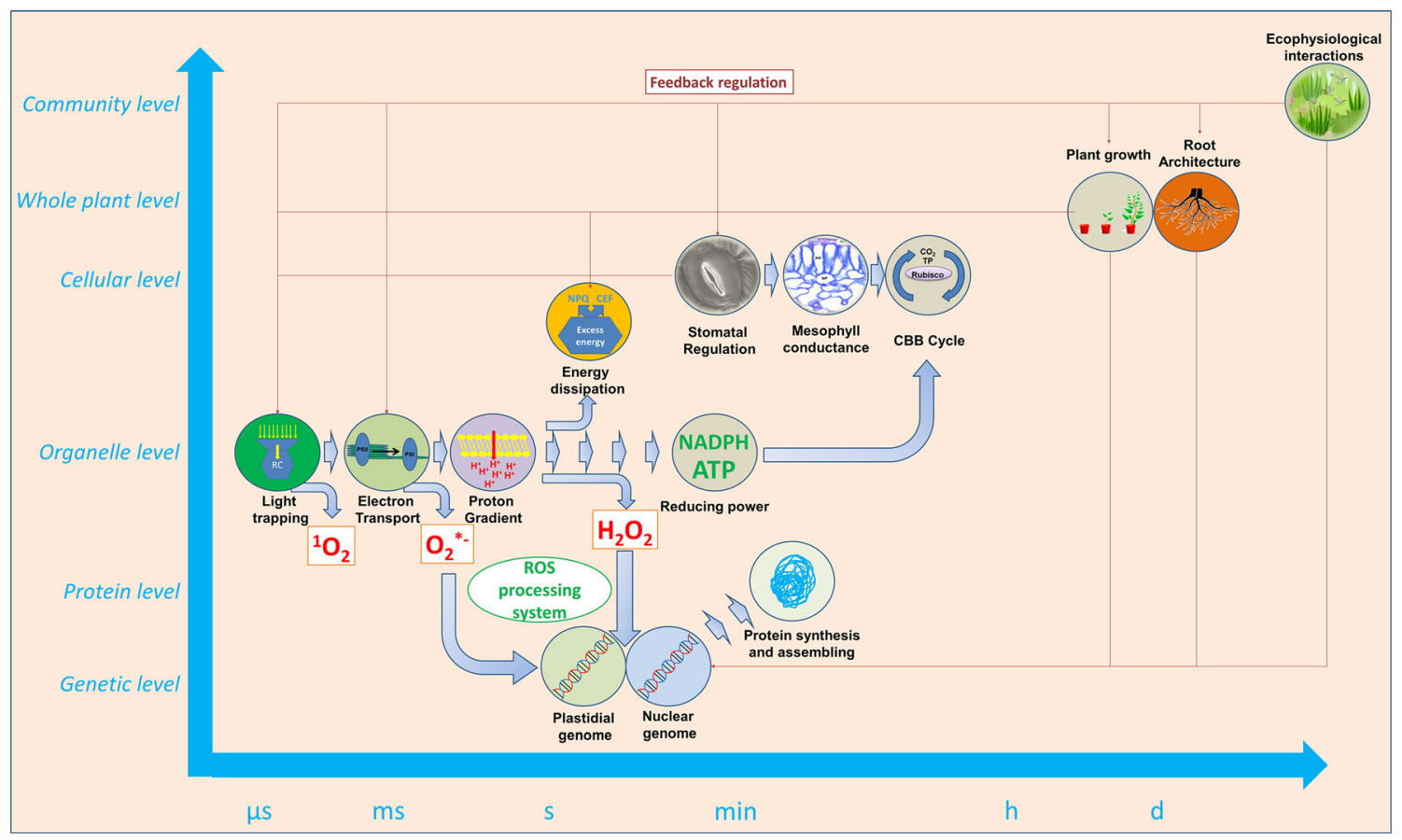

Fig. 1 View of photosynthesis as an emergent and non-linear process, with complex properties separated in space-time. The onset of photosynthesis is determined by the biophysics events related with the light trapping and charge separation in the photosystems, which occurs in the complex of proteins and pigments assembled in the thylakoid membranes (organelle level) and in the time-scale of microseconds or lesser. Electron transport, proton gradient generation, NPQ induction and ROS signalling events are separated in a time-frame from milliseconds to few minutes. These processes are crucial to the regulation of gene expression in both plastidial and nuclear genome levels, which may lead to changes in proteomic profile in a time-scale of few minutes to hours. The reductive phase of photosynthesis is activated much later in a time-frame of minutes to hours (cellular level) and, ultimately, is able to produce the carbon skeletons needed to shoot and root growth and morphology (whole plant levels) in a time frame of few days to several years. Remarkably, all these processes are non-linear and a strong feedback regulation effect is involved in the integrated responses between the upper-scale levels with those more basic, which is incompatible with simple direct causeeffect relationships 
can exhibit different responses to a same external condition (Vítolo et al. 2012; Bertolli et al. 2014; Amaral and Souza 2017).

It is remarkable that the photosynthetic apparatus's activity and composition and its acclimatization to the fluctuating environment are self-regulated (by the way, as all other plant processes are). Products and intermediate metabolites are regulated through feedback and feed-forward loops, controlling the operation of both photochemical and $\mathrm{CO}_{2}$ assimilation reactions (e.g., Anderson et al., 1995). Perhaps due to these systemic features, the dynamics properties and scaling rules of the photosynthesis processes, as a complex system, remain unclear (Souza et al. 2016b). Indeed, studying the mechanisms underlying the variation of photosynthetic capacity in a spatial-temporal context can raise an interesting question: how could these cellular processes be extrapolated to a whole individual or to a population under a natural environment? Actually, plants often exhibit very plastic responses to changing environmental factors such as light, water, nutrients, and temperature, among others. These responses to oscillations of external factors have been fine-tuned by billions of years under natural selection, and it is subjected to genetic control and regulation shaped by the native environments of plant ancestors in order to reach whole system stability more than getting maximum use efficiency of the resources (Souza and Lüttge, 2015; Arp et al. 2020).

\section{Non-photochemical quenching mechanism as an example of photosynthetic complexity and emergence}

Non-photochemical quenching (NPQ) can be seen as a concrete example of how reductionist and simplistic approaches have led to controversial debates in the study of photosynthesis. Although much knowledge has been acquired from biophysical studies associated with the use of mutants transformed for protein deficiencies in the PSII antenna complexes and enzymes associated with xanthophyll synthesis, the proper site of qE (energy component of NPQ) formation is still scene of a heated debate in the literature (Murchie and Ruban 2020). While some groups have argued regarding the importance of certain chlorophyll or lutein molecules positioned at special points of certain subunits of photosystem II antennae, transformed plants have showed contradictory results and no one statement has been able to fully explain the right place where that process occurs, as well as the player components and the exact biophysical mechanisms of this simple photosynthetic process (Ruban et al. 2012).

Interestingly, recent studies involving the combination of two approaches, a semi-empirical method for quantum calculation of molecular electronic structure (MNDO-CAS-CI), and the transition density cube (TDC) technique for calculating electronic coupling strengths between molecules, have proposed a new model for all chlorophyll-carotenoid energy transfer pathway in highly quenched LHCII X-ray structure (Chmeliov et al. 2015). The main conclusions that can be drawn from this study involving computational chemistry and mathematical modelling is the prospect of understanding $\mathrm{qE}$ not only as a process caused by a single pair or small group of pigments localized in PSII antennas, but as the result of a complete systematic re-arrangement involving several chlorophyll and carotenoid pigments associated with a few dozen proteins from this small sub-component module of the whole photosynthetic system. This example involving NPQ is emblematic because it reveals that even at the most basic hierarchical levels of photosynthesis, the systemic view can provide a more realistic alternative than the simplistic classic reductionist alternative.

However, still using the same NPQ example, although relative progress has been achieved in exploring the individual modules of photosynthesis, in some circumstances even adopting a more systemic view on the sub-components, the crucial integration of the knowledge obtained in these sub-modules with the different upper-scale levels that define the plant system as a whole, has not been accomplished yet. We need to study the NPQ process, for instance, far beyond a "basic" assembling of a set of pigments and proteins leading to biophysical consequences for dissipation of excess energy in the form of heat in chloroplasts. In fact, each PSII antenna is inserted into the thylakoid membrane and associated with a few dozen other proteins and super-complexes. This complex structure is committed to the synthesis of ATP and NADPH, affecting the $\mathrm{pH}$ and redox environment of chloroplasts. These changes have crucial consequences on the biochemical and molecular arrangement of the PSII's antennas and therefore, 
presenting potential feedback effects on the NPQ process.

Those phenomena are beautiful and typical examples of "emergence propriety" in plants as preconized by the systems theory. According to Goldstein (2002), emergence accounts for "the arising of novel and coherent structures, patterns and properties during the process of self-organization in complex systems". In other words, emergent properties are those not observed in the components (or subsystems) of a particular system that is under observation. For instance, while a gas inside a closed bottle exhibits physical properties such as temperature and pressure, each individual atom that constitutes that gas has neither pressure nor temperature. Thus, pressure and temperature are emergent properties that "pop up" while many atoms constituent of a particular gas interact under a specific context (e.g., a bottle at room conditions). On the other hand, modularity is a reductionist approach by which a system's feature are supposed to result from its structural and functional parts that act more or less semi-autonomously (Lüttge 2012). Therefore, a system could be characterized by studying its individual components, or 'modules', in a linear fashion, supposing straightforward causal (deterministic) relationships between different parts (Wegner 2019), which is the predominant approach in the mainstream of photosynthesis research. Even the current claim for a "system biology" perspective (Mochida and Shinozaki 2011; Sheth and Thaker 2014) is passive of criticism since systems biology, with its mostly bottom-up approach, deals with plant as an "average" assembly (or, rather, by-product) of molecular 'modules' and their mechanistic interaction. As pointed out by Lüttge (2021), these concepts tend to undervalue and neglect synergistic emergence as a key feature of living organisms.

Moreover, although the information obtained through in vivo measurements of chlorophyll $a$ fluorescence has contributed to understanding several photosynthetic processes, including NPQ, the biochemical and molecular mechanisms are still assessed mainly through destructive analytical techniques. These approaches include, for example, the maceration of plant tissues for the extraction of pigments, proteins, nucleic acids, and other metabolites and, consequently, the measurement of these components by in vitro tests, often under optimal conditions, utterly different from that found in a fully functioning plant cell. For instance, it is assumed that all cells in a tissue sample are mostly in the same steady-state physiological condition, which is an obvious oversimplification (Long et al. 2020). Worse, often due to sampling limitations, the time factor is neglected, making the results, at best, more putative than realistic. In this vein, the investigators commonly try to establish correlations between photochemical efficiency and the amount of D1 protein without considering that these analytical methods are entirely separated by the dynamic balance and spatial heterogeneity that occur inside the plant cell. These limitations certainly add many layers of complexity to a more accurate and realistic interpretation of experiments involving photosynthesis and should not be simply overlooked by the researchers.

Indeed, a plethora of complexity levels may be added when considering the upper scales of plant organization influencing the more basal modules. For example, the species-specific differences in mesophyll conductance may be directly associated with greater or lesser consumption of reducing power, which ultimately will reflect in the photochemical process, including NPQ (Coopman et al. 2011). How could be this far photosynthetic process perfectly integrated with NPQ in each plant stress experiment? Thus, it is urgent to think about the consequences of the whole growth rates per se also affected by the environment, such as water availability and nutrients in the soil and how this entire balance could reflect in upstream photosynthetic processes as NPQ and photoinhibition, and vice versa. Therefore, to understand a phenomenon as complex as photosynthesis, the adoption of a simplistic reductionist view cannot be justified anymore, yet some specific missing details at molecular levels are worth to be uncovered.

\section{How complex are the interactions regulating the photosynthetic efficiency in different species, individuals and environments?}

Photosynthetic efficiency emerges from an integrative network of many plant characteristics. Commonly, the most effective plant is assumed to present higher photosynthetic rates, which could be a massive simplification of reality. Thus, considering the complex interactions between plants and their environments, especially those factors that might determine 
the lifetime plant performance, other features also should have to be considered. The "optimization assumption" of natural systems has been severely criticized as an anthropomorphic concept. Based on that, different questions can emerge: What is most important: to be the best or to be more effective? Are plants designed to maximize their carbon gain? Wouldn't be stability the main "goal" of evolutionary process than optimality, especially considering the everlasting changing environment?

Another important concern is regarding the current experimental approaches employed in photosynthesis studies. Often experiments consist of individual model plants isolated from their natural populations and rarely integrative responses are evaluated. Thus, the characteristics that maximize the individual plant's fitness in these experiments are broadly distinct from their natural environment conditions. Indeed, plants commonly grow near together and are strongly influenced by the foraging competition for light, water and nutrients, among other abiotic and biotic factors. Assuming that the individual's performance is independent of the characteristics of its neighbours is, in fact, ignoring the competitive/collaborative interactions between plants and other organisms (Heil and Karban 2010; Sharifi and Ryu 2020). Hence, a longstanding challenge in plant biology is: how much confidence do we have to extrapolate our experimental conclusions from individual plants to the vegetation's structure in its natural environment? This question is undoubtedly crucial, but not the unique limitation to escape from the simplistic view of photosynthesis.

Time is another critical issue. Photosynthesis is commonly studied at one stead-state point in time, ignoring the dynamics of growth and the dramatic changes in time that this process exhibit, which are crucial in comprehending the differences between dynamic and steady-state responses (Fig. 2). As modulated systems, plants may present photosynthetic responses at the photochemical phase in a scale of milliseconds, a response at stomatal level in a few minutes and several changes in the expression, translation and assembling of proteins in chloroplasts by few hours (Fig. 1). Linearly, it is apparently easy to understand, but as the time lapse, these different regulation levels may affect each other simultaneously and reciprocally. Indeed, the temporal steady-state dynamics of photosynthesis can reach highly complex non-linear behaviours, like many other physiological processes (Hütt and Lüttge 2002; Kaiser et al. 2015). Souza et al. (2009) have observed chaotic-like dynamics on leaf gas exchanges even under nondisturbed and constant external conditions. More interestingly, it was supposed a positive relationship between complexity and stability. This imply that more irregular gas exchanges enable plants to cope more effectively with external disturbances, providing more flexibility for physiological adjustments, which were related to higher level of network connectance (i.e., tighter network) among different variables underlying leaf gas exchanges (Souza et al. 2009).

The results obtained from in vivo conditions represent the steady-state balance of all these dynamic processes in response to the environmental stimuli throughout the plant lifetime (see Figs. 1 and 2). So, could we assume that the plants presenting higher photosynthetic capacity should have the best lifetime performance? The answer to this question is not straightforward. For instance, a well-studied trade-off is assumed occurring between photosynthetic rates and leaf longevity. Leaves with low photosynthetic capacity commonly have higher longevity than the ones with higher photosynthetic rates (Coley 1988). This mentioned trade-off is related to nitrogen availability, one of the many factors that can limit the photosynthetic efficiency. Photosynthesis capacity depends not only on how nitrogen is distributed among leaves but also how it is partitioned on the leaf area's total amount. The photosynthetic nitrogen use efficiency (PNUE), a ratio between photosynthetic performance per $\mathrm{N}$ content, varies among $\mathrm{C} 3$ species and this variation emerges from an integration of different features in a complex network.

The photosynthetic parameter, PNUE, might induce difficulties for a correct interpretation, since it is indicating a ratio between two interdependent and complex processes, $\mathrm{CO}_{2}$ assimilation and leaf $\mathrm{N}$ acquisition. For instance, some authors have postulated that less nitrogen is allocated to Rubisco in lowPNUE species (Feng et al. 2007), but this is not an unequivocal rule. Stomatal and mesophyll conductance plays a significant role in the variation of PNUE, but their effects may be different among species. It has been suggested that the cell wall could be an important sink to $\mathrm{N}$ and its thickness in mesophyll cells could affect the mesophyll conductance (Evans et al. 2009). In addition, plants increase $\mathrm{CO}_{2}$ uptake by assimilating nitrogen via the photorespiratory pathway (Busch 

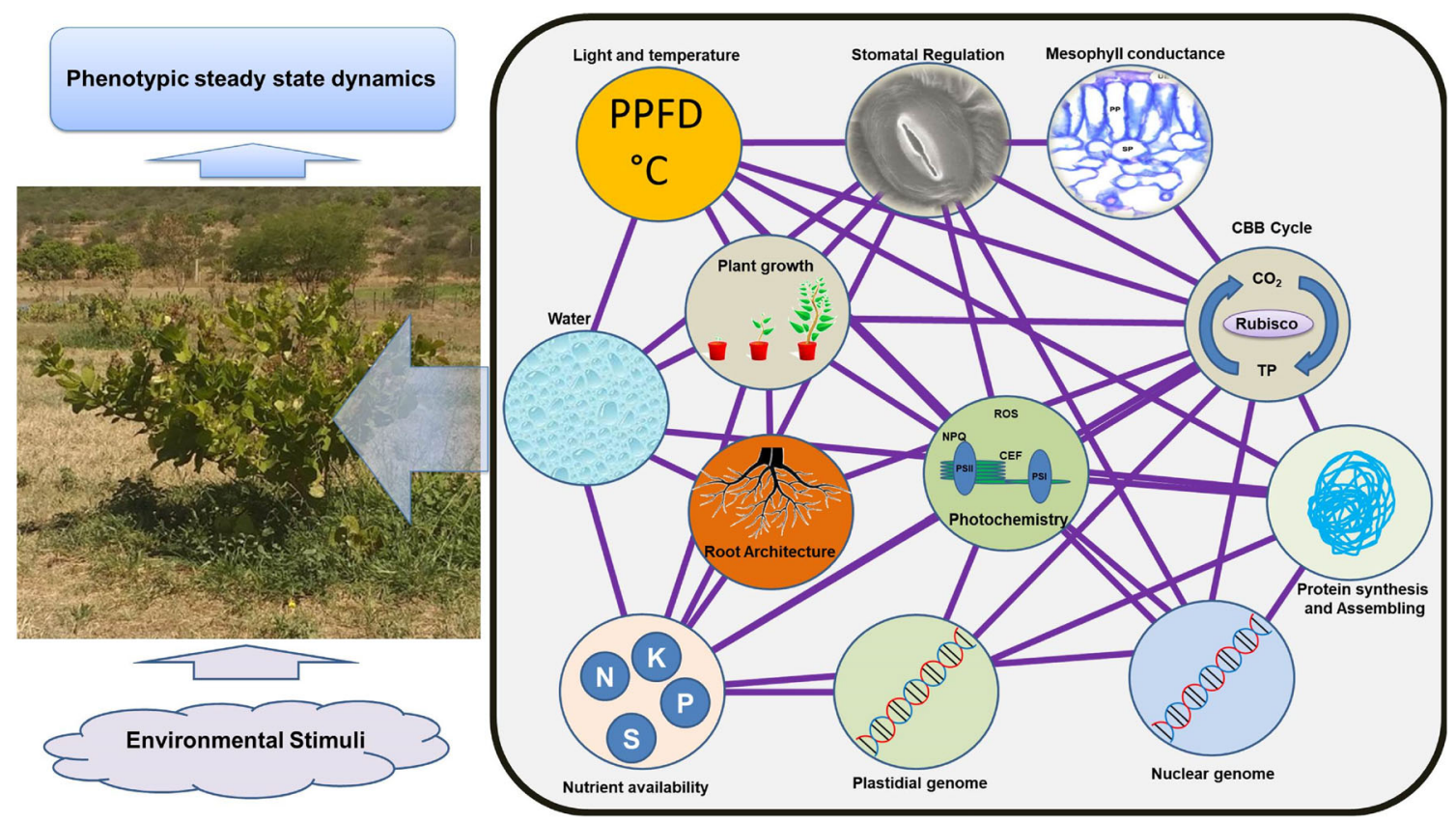

Fig. 2 Photosynthesis as a non-linear component, decentralized and complex process in plant systems. The initial environmental stimuli, which could be generated by different abiotic and biotic stimuli, such as water and nutrient availability, temperature and others, is processed by plant systems as a non-linear and decentralized information. Consequently, photosynthesis, as well as gene expression regulation and the whole plant growth

et al. 2018) and $\mathrm{N}$ metabolism can produce several secondary $\mathrm{N}$-metabolites and these pathways can be plant species dependent. Thus, such interspecific variations in nitrogen allocation to other functions may weaken the correlation between Rubisco and PNUE and add an extra layer of complexity in understanding photosynthesis in plants.

\section{The plant species matter: photoinhibition as a specific and complex mechanism for photosynthesis efficiency}

Based on all this evidence discussed previously, a critical assumption must be taken here: the plant species matter! However, most of our knowledge related to photosynthesis have emerged from studies in Arabidopsis thaliana and few other crops such as rice, tobacco and maize. This limitation launches a huge shadow on the understanding of the complexity of photosynthetic process in plant realm, especially on these species that are growing in uncommon and morphology are integrated as unique big event, with strong feedback regulatory effects. According to this systemic view of photosynthesis, the simplistic cause-effect relationships are not possible and could not be employed to understand the phenotypic steady state dynamics of a given plant in response to a specific combination of environmental factors, in a given moment in the plant lifetime

environments. The understanding of photoinhibition as a central photosynthetic mechanism for plant protection and/or reflecting a stressful condition, is an emblematic example of such a problem. Photoinhibition on PSII is directly related to D1 protein damage and recovery. The capacity for a fast turnover rate of D1 and the ability to repair and reuse damaged PSII depends on several factors (Lima Neto et al. 2017; Cerqueira et al. 2019). Since most traditional knowledge about PSII photoinhibition is based on studies involving model species, it is mostly assumed that photoinhibition intensity is a stress marker.

For many years plant breeders had searched for low photoinhibition levels in plants as a useful marker for inferring plant resistance against stress. However, again, this is not the plant's real world. In nature, several plants, especially those known as "evergreen species", may induce a strong stomatal closure in response to drought, salt or cold stimuli. This process leads to an unbalance between energetic demand and energy capture. In such cases, some plants may induce a robust energy dissipative processes, as already 
previously discussed, which may protect the plants from excess energy for days. An interesting example of this regulation may be observed in cashew plants (Anacardium occidentale), a semiarid adapted species, which are able to modulate the differences phases of photosynthesis in parallel to the induction of a strong antioxidant defence system. Due to such adjustments, cashew plants may face extreme abiotic adverse conditions without any change in PSII integrity (Lima et al. 2018).

The maintaining of photochemical integrity in plants is highly complex and non-linear since the photosystems do not function isolated but integrated in an orchestrated system. For instance, the recovery of plants from PSI photoinhibition is relatively slow and the duration of reduced photosynthetic capacity from PSI photoinhibition is longer than that from PSII photoinhibition, which is likely to have a severe negative impact on the photosynthetic efficiency (Cerqueira et al. 2019). Previous studies have shown that the oxidation of $\mathrm{P} 700$ provides protection from PSI photoinhibition, reducing the formation of superoxide radicals and singlet oxygen (Sejima et al. 2014). Interestingly, previous results have evidenced that PSI photoinhibition activity in response to high light might trigger antioxidant defenses to photosynthesis protection by mechanism unknown yet (Lima-Melo et al. 2019a, b).

Recent studies have shown that photosynthetic organisms from cyanobacteria to land plants are able to display a myriad of different mechanisms to stimulate the oxidation of P700 (Takagi et al. 2017). In cyanobacteria, the overreduction state of PQ-pool decreases Q-cycle turnover at the Cytb6f level, and this event results in suppressing electron flow to the PSI (Shaku et al. 2016). In addition, flavodiiron proteins (Flv) might release electrons from PSI in cyanobacteria (Shimakawa et al. 2016). Recently, these proteins were discovered as important in ferns and gymnosperms inducing rapid oxidation of P700 in presence of an over-reduced photosynthetic electron transport chain (Takagi et al. 2017). On the other hand, angiosperms are capable to oxidize P700 dependent on $\mathrm{H}^{+}$-gradient formation across the thylakoid membranes, stimulating photorespiration when the CalvinBenson-Bassham cycle activity is suppressed.

A recent study with Jatropha curcas, a wild species well-adapted to cope with extreme hot and dry conditions, showed that the activation of the cyclic electron flow is an important photoprotective mechanism avoiding the excess energy in both photosystems (Lima Neto et al. 2017). Under circumstances of excess energy on thylakoids, the maintenance of P700 in an oxidized state is an important mechanism to avoid ROS burst in this species (Cerqueira et al. 2019). Also, upon inhibition of PSI, the highly reduced electron transport chain triggers a significant increase in $\mathrm{H}_{2} \mathrm{O}_{2}$ content, promoting PSI photoinhibition. In contrast, Ricinus communis, another wild species welladapted to semiarid environments, do not trigger CEF efficiently in response to excess energy. This species strongly increases photorespiration as a photoprotective mechanism under drought (Lima Neto et al. 2017). However, the downregulation of photorespiration under optimum growth condition did not impact on $R$. communis growth, but under excess energy its inhibition promoted oxidative stress with consequences on photosynthetic efficiency (Ziotti et al. 2019). Thus, it was clearly remarked here that plant species display different mechanisms to manage the excess energy and then regulate the photosynthetic efficiency.

Many efforts have been made to increase photosynthetic efficiency such as screening of natural diversity of Rubisco structure and kinetic properties. Model analysis suggesting that the replacement of native Rubisco for other displaying improved kinetic properties has been postulated for increases in photosynthesis in future climate conditions (McGrath and Long 2014). Additionally, photorespiration bypass by engineering of $\mathrm{C} 3$ plants seems promising. However, some used methods were shown to disturb NADH supply to mitochondria and therefore can be counterproductive (Betti et al. 2016). Improving C3 plant photosynthesis by introducing carbon-concentrating mechanisms by diffusion facilitation, including increase in carbonic anhydrase activity, have been also suggested as a possible target for improving photosynthesis. Nevertheless, due to the complexity of the process, the progress on changing these specific targets to increase photosynthetic efficiency has been a laborious and difficult activity. A recent study tested the hypothesis that overexpression of CA1P phosphatase would stimulate Rubisco's activity and photosynthesis by decreasing the amount of CA1P, a modulator of Rubisco's activity, in wheat leaves. The controversial and surprising outcome was that CA1P overexpression resulted in low Rubisco abundance 
and photosynthesis (Lobo et al. 2019), showing again the complexity of the plant system.

Therefore, it is essential to understand that our current and common scientific sense of reductionism is perfunctory in terms of the complexity and integrated network regulating photosynthetic process. Using the current approaches and experimental models it is difficult to synthesize, or put the parts together, to solve the whole photosynthetic performance, and most importantly, to produce robust conclusion and extrapolations. However, these are the tools that currently can provide us with some feasible discoveries in this field. It is crucial and prudent to understand how far we can achieve our conclusions in an assertive, powerful and integrated way. These observations make us reflect and glimpse on the fact that a vast new world of possibilities is still to be discovered on photosynthesis research.

\section{The plant as a whole complex and multi-scale organism}

Putting the things in straight lines: considering plants as a mere assemble of semi-automatous parts, driven by deterministic mechanical rules, constituting a purely reactive organic system should be no-longer the mainstream perspective on XXI Century and forward. This classical scientific approach, as we stressed before, allow us to uncover many fundamental details on what plants are made of, and some basic aspects of local integration, that is, we have huge amount of information about what constitute a plant, some understanding about pivotal physiological functions, yet few knowledge on what plants are at all and how they actually interact with the complexity surrounding them. Likely, the main problem to adopt a pure classical mechanistic view of living beings is the misconception that straightforward causal (deterministic) relations between components of a biological system components represents accordingly the whole complexity of a living organism. Actually, in such systems, are dominant non-linear and unpredictable dynamic networks, especially when trying to scale up cross-systems hierarchical organization. Certainly, that eventual deterministic relationship in lower-level subsystems is reliable to be found in a consistent way (e.g., the role of specific genes regulating monogenic traits). But, considering the plant as whole, that's the exception more than the general rule.

So, what options do we have besides these current approaches? A much more difficult task than determining our limitations is proposing solutions to such problems. The definitive solution, although is not still available, should involve adopting a more critical and less simplistic stance in relation to the results obtained currently in photosynthesis studies. These relations are, most probably, do not feasible to understand photosynthetic metabolism, and much less yet to evaluate organization and responses displayed by complex systems. In many cases, plants transformed for the expression of a single gene do not show statistically significant changes at the photosynthetic level but can exhibit changes in other hierarchical levels that might include the transcriptome, proteome and metabolome responses (Carvalho et al. 2014; Cunha et al. 2019). Therefore, knowing the great complexity and, above all, the high rate of metabolic redundancy and functional degeneration of the plant systems, we cannot jump directly to hasty conclusions without first examining some of these crucial emergent properties. Changes in more basic hierarchical levels, associated with high plasticity and activation of redundant and compensatory mechanisms in plant cells, may be able to well dispel the influence of a given genetic product deficiency on the photosynthetic process as a whole, without, however, proving that it does not exist under natural conditions (Souza et al. 2016a, b).

In this sense, the parallel use of different omics approaches can represent an excellent possibility for understanding photosynthesis in a more systemically way. In fact, all the omics approaches available today are related to destructive techniques, therefore, not close enough to plants' real world. However, when these techniques are combined with morpho-anatomical, biophysical (in vivo) and reverse genetic approaches, this robust set of information can be useful to provide a more systemic view of what actually occurs in relation to the photosynthetic process a given environmental condition. Logically, the experimental design that involves all these components must be well-planned and precludes the fluctuations of variables in response to space-time changes. Unfortunately, although many studies have been carried out involving all these approaches and concepts mentioned here, only a few integrate all of 
this and attempt to contribute for significant interpretation and capacity for a robust generalization. Furthermore, most omics studies, for example, have the technique as their main purpose and are limited to the generation of a vast data volume that is commonly very difficult to reproduce, denying any possibility of in-depth physiological interpretation.

The use of network approaches, which can be based on Pearson's correlation levels or measurements of physical and chemical interactions, are also good alternatives to try to step back and analyse the biological problem from a holistic and more real perspective (Souza et al. 2009). Through the use of these approaches, which are increasingly accessible through the use of specialized software and powerful modern tools like artificial intelligent, that make the work considerably reduced, parameters such as global connectivity and the clustering coefficient can be easily estimated (Guilherme et al. 2019). These parameters are example of tools that can be promptly used to see the systemic changes underlying any photosynthetic responses to a given stimulus, such as molecular silencing of a gene or exposure to adverse environmental conditions. This will certainly be a hot topic in the near future related to photosynthesis research.

\section{Concluding remarks and perspectives}

In view of what was exposed in this critical opinion/ review, it becomes increasingly evident that the traditional reductionist view, associated with simplistic approaches, presents an important concern for a most complete understanding on photosynthesis. These scientific findings preclude superficial direct cause-and-effect relationships, encompassing a nonintegrative and centralized view of the various modules that make up the photosynthetic process, but they are insufficient to understand how global photosynthesis works. This problem is aggravated by excessive centralization in Arabidopsis studies and few other species, neglecting studies in non-model species. Besides other important difficulties such as the current predominance of simplistic and artificial experimental conditions, in which, altogether, constitute a significant limitation for understanding photosynthesis in plants. Therefore, the need for reinforce systemic and integrative perspectives, capable of understanding plants as complex, redundant and self-regulating networks they are, is crucial.

The use of combined approaches, including biophysics, biochemistry, molecular biology and omics, among others, especially in the view of steady-state dynamics in respect of space-time changes, can be of extreme importance (Fig. 2). Therefore, we believe that photosynthesis research, as plant physiology, is entering in a new era, in which the relationships and synthesis from function of isolated parts, especially under real conditions, become crucial. The results obtained by using different methods could be synergistically combined with new methods still to come. We suggest here that this transition will be of great importance for the future of research in photosynthesis, especially for obtaining more resistant and productive cultivars in an imminent climate changing scenario, and the research on photosynthesis will remain firmly at the forefront of biological science for a long time to come.

Ultimately, it is imperative to emphasize that as we advance our knowledge about the photosynthetic process, it becomes even more evident that understanding this phenomenon will not result from the effort of a single field of science. The need to think systemically about plants requires that the plant physiologist of the twenty-first century also functions as a "module" integrated to a "large scientific network of knowledge". Associations among biologists, agronomists, physicists, mathematicians and computer scientists, for example, will be crucial to give a biological and practical meaning to the large amount of information that is generated by thousands of papers that are produced annually. Only then we will be able to delve deeply into the characteristic aspects of each of the modules that make up the photosynthesis process, but also to take a step back and understand photosynthesis in its systemic context in the plant as a whole. This knowledge will undoubtedly be essential for future generations to take the correct steps in guaranteeing food security and increasing the efficiency of land use, especially in an increasingly worrying climate change scenario.

Acknowledgements We are thankful to the Brazilian Society of Plant Physiology for supporting the I-BSP, the organizing and scientific committee of the symposium for the effort and commitment to this idea, and to all guest speakers and participants. 
Funding This work is supported by grant \#2018/04258-6, São Paulo Research Foundation (FAPESP), grant \#404707/2018-1 CNPq, INCT Plant Stress Biotech (Conselho de Desenvolvimento Científico e Tecnológico-CNPq) Proc. 465480/2014-4 and Fundação Cearense de Apoio ao Desenvolvimento Científico e Tecnológico (FUNCAP)—FCT00141-00010.01.00/18. We also acknowledge CNPq for the financial support (Grant 428192/2018-1) and the research fellowship (\#302715/2018-5 CNPQ) granted to GMS. FELC was supported (2018-2020) by FUNCAP/CAPES (CAPES/ BRASIL-Proc. 88887.162856/2018-00) and is currently supported by Corporación Colombiana de Investigación Agropecuaria - Agrosavia.

Data availability Data sharing not applicable to this article as no datasets were generated or analysed during the current study.

\section{Declaration}

Conflict of interest The authors declare that they have no competing interest.

\section{References}

Amaral MN, Souza GM (2017) The challenge to translate OMICS data to whole plant physiology: the context matters. Front Plant Sci 8:2146. https://doi.org/10.3389/fpls. 2017.02146

Anderson JM, Chow WS, Park YI (1995) The grand design of photosynthesis: acclimation of the photosynthetic apparatus to environmental cues. Photosyn Res 46:129-139

Arp TB, Kistner-Morris J, Aji V, van Cogdell RJ, Grondelle R, Gabor NM (2020) Quieting a noisy antenna reproduces photosynthetic light-harvesting spectra. Science 368(6498):1490-1495

Betti M, Bauwe H, Busch FA, Fernie AR, Keech AR, Levey M, Ort DR, Parry MAJ, Sage R, Timm S, Walker B, Weber APM (2016) Manipulating photorespiration to increase plant productivity: recent advances and perspectives for crop improvement. J Exp Bot 67:2977-2988. https://doi. org/10.1093/jxb/erw076

Bertolli SC, Souza GM (2013) The level of environmental noise affects the physiological performance of Glycine max under water deficit. Theor Exp Plant Physiol 25:36-45

Bertolli SC, Mazzafera P, Souza GM (2014) Why is it so difficult to identify a single indicator of water stress in plants? A proposal for a multivariate analysis to access emergent properties. Plant Biol 16:578-585

Busch FA, Sage RF, Farquhar GD (2018) Plants increase $\mathrm{CO}_{2}$ uptake by assimilating nitrogen via the photorespiratory pathway. Nat Plants 4:46-54. https://doi.org/10.1038/ s41477-017-0065-x

Carvalho FEL, Ribeiro CW, Martins MO, Bonifacio A, Staats CC, Andrade CMB, Cerqueira JV, Margis-Pinheiro M, Silveira JAG (2014) Cytosolic APX knockdown rice plants sustain photosynthesis by regulation of protein expression related to photochemistry, Calvin cycle and photorespiration. Physiol Plant 150(4):632-645
Cerqueira JVA, Silveira JAG, Carvalho FEL, Cunha JR, Lima Neto MC (2019) The regulation of P700 is an important photoprotective mechanism to $\mathrm{NaCl}$-salinity in Jatropha curcas. Physiol Plant 167(3):404-417. https://doi.org/10. 1111/ppl.12908

Chmeliov J, Bricker WP, Lo C et al (2015) An "all pigment" model of excitation quenching in LHCII. Phys Chem Chem Phys 17:15857-15867. https://doi.org/10.1039/ C5CP01905B

Coley PD (1988) Effects of plant growth-rate and leaf lifetime on the amount and type of plant anti-herbivore defence. Oecologia 74:531-536

Coopman RE, Briceno VF, Corcuera LJ, Reyes-Diaz M, Alvarez D, Saez K, Garcia-Plazaola JI, Alberdi M, Bravo LA (2011) Tree size and light availability increase photochemical instead of non-photochemical capacities of Nothofagus nitida trees growing in an evergreen temperate rain forest. Tree Physiol 31(10):1128-1141

Cunha JR, Carvalho FEL, Lima-Neto MC et al (2019) Proteomic and physiological approaches reveal new insights for uncover the role of rice thylakoidal APX in response to drought stress. J Proteomics 192:125-136. https://doi.org/ 10.1016/j.jprot.2018.08.014

Evans JR, Kaldenhoff R, Genty B, Terashima I (2009) Resistances along the $\mathrm{CO}_{2}$ diffusion pathway inside leaves. J Exp Bot 60:2235-2248

Feng YL, Auge H, Ebeling SK (2007) Invasive Buddleja davidii allocates more nitrogen to its photosynthetic machinery than five native woody species. Oecologia 153:501-510

Goldstein J (2002) The singular nature of emergent levels: suggestions for a theory of emergence. Nonlinear Dyn Psychol Life Sci 6:293-309

Guilherme EA, Nascimento CS, Lobo AKM et al (2019) Nitrogen-utilization efficiency during early deficiency after a luxury consumption is improved by sustaining nitrate reductase activity and photosynthesis in cotton plants. Plant Soil 443:185-198. https://doi.org/10.1007/ s11104-019-04214-7

Heil M, Karban R (2010) Explaining evolution of plant communication by airborne signals. Trends Ecol Evol 25(3):137-144

Hütt M-T, Lüttge U (2002) Nonlinear dynamics as a tool for modeling in plant physiology. Plant Biol 4:281-297

Kaiser E, Morales A, Harbinson J, Kromdijk J, Heuvelink Ep, Marcelis LFM (2015) Dynamic photosynthesis in different environmental conditions. J Exp Bot 66(9):2415-2426

Li J, Wang M, Li Y et al (2019) Multi-omics analyses reveal epigenomics basis for cotton somatic embryogenesis through successive regeneration acclimation process. Plant Biotechnol J 17:435-450. https://doi.org/10.1111/pbi. 12988

Lima CS, Ferreira-Silva SL, Carvalho FEL et al (2018) Antioxidant protection and PSII regulation mitigate photooxidative stress induced by drought followed by high light in cashew plants. Environ Exp Bot 149:59-69. https://doi. org/10.1016/j.envexpbot.2018.02.001

Lima-Melo Y, Gollan P, Tikkanen M, Silveira JAG, Aro EM (2019a) Consequences of photosystem I damage and repair on photosynthesis and carbon use in Arabidopsis thaliana. Plant J 97:1061-1072 
Lima-Melo Y, Alencar VTCB, Lobo AM, Sousa RHV, Tikkanen M, Aro EM, Silveira JAG (2019b) Photoinhibition of PSI provides oxidative protection during imbalanced photosunthetic electron transport in Arabidopsis thaliana. Front Plant Sci 10:1-13

Lima-Neto MC, Cerqueira JVA, Cunha JR et al (2017) Cyclic electron flow, NPQ and photorespiration are crucial for the establishment of young plants of Ricinus communis and Jatropha curcas exposed to drought. Plant Biol 19:650-659. https://doi.org/10.1111/plb.12573

Lobo AKM, Orr DJ, Gutierrez MO, Andralojc PJ, Sparks C, Parry MA, Carmo-Silva E (2019) Overexpression of ca1pase decreases rubisco abundance and grain yield in wheat. Plant Physiol 181(2):471-479

Long Y, Cheddadi I, Mosca G, Mirabet V, Dumond M, Kiss A, Traas J, Godin C, Boudaoud A (2020) Cellular heterogeneity in pressure and growth emerges from tissue topology and geometry. Curr Biol 30(8):1504-1516

Lüttge U (2012) Modularity and emergence: biology's challenge in understanding life. Plant Biol 14:865-871

Lüttge U (2021) Integrative emergence in contrast to separating modularity in plant biology: views on systems biology with information, signals and memory at scalar levels from molecules to the biosphere. Exp Plant Physiol, Theor. https://doi.org/10.1007/s40626-021-00198-x

McGrath JM, Long SP (2014) Can the cyanobacterial carbonconcentrating mechanism increase photosynthesis in crop species? A theoretical analysis. Plant Physiol 164(4):2247-2261

Mochida K, Shinozaki K (2011) Advances in omics and bioinformatics tools for systems analyses of plant functions. Plant Cell Physiol 52:2017-2038. https://doi.org/10.1093/ $\mathrm{pcp} / \mathrm{pc}$

Murchie EH, Ruban AV (2020) Dynamic non-photochemical quenching in plants: from molecular mechanism to productivity. Plant J 101:885-896. https://doi.org/10.1111/tpj. 14601

Nelson N, Ben-Shem A (2004) The complex architecture of oxygenic photosynthesis. Nat Rev Mol Cell Biol 5(12):971-982. https://doi.org/10.1038/nrm1525

Ruban AV, Johnson MP, Duffy CDPP (2012) The photoprotective molecular switch in the photosystem II antenna. Biochim Biophys Acta Bioenerg 1817:167-181. https:// doi.org/10.1016/j.bbabio.2011.04.007

Sejima T, Takagi D, Fukayama H, Makino A, Miyake C (2014) Repetitive short-pulse light mainly inactivates photosystem I in sunflower leaves. Plant Cell Physiol 55:1184-1193

Shaku K, Shimakawa G, Hashiguchi M, Miyake C (2016) Reduction-induced suppression of electron flow (RISE) in the photosynthetic electron transport system of Synechococcus elongatus PCC 7942. Plant Cell Physiol 57:1443-1453

Sharifi R, Ryu CM (2020) Social networking in crop plants: wired and wireless cross-plant communications. Plant Cell Environ. https://doi.org/10.1111/pce.13966
Sheth BP, Thaker VS (2014) Plant systems biology: insights, advances and challenges. Planta 240:33-54. https://doi. org/10.1007/s00425-014-2059-5

Shimakawa G, Shaku K, Miyake C (2016) Oxidation of P700 in photosystem I is essential for the growth of cyanobacteria. Plant Physiol 172:1443-1450

Silveira JAG, Carvalho FEL (2016) Proteomics, photosynthesis and salt resistance in crops: an integrative view. J Proteomics 143:24-35. https://doi.org/10.1016/j.jprot.2016. 03.013

Sousa RHV, Carvalho FEL, Lima-Melo Y et al (2019) Impairment of peroxisomal APX and CAT activities increases protection of photosynthesis under oxidative stress. J Exp Bot 70:627-639. https://doi.org/10.1093/jxb/ery354

Souza GM, Ribeiro RV, Prado CHBA et al (2009) Using network connectance and autonomy analyses to uncover patterns of photosynthetic responses in tropical woody species. Ecol Complex 6:15-26. https://doi.org/10.1016/j. ecocom.2008.10.002

Souza GM, Lüttge U (2015) Stability as a phenomenon emergent from plasticity-complexity-di- versity in eco-physiology. In: Lüttge U, Beyschlag W (eds) Progress in botany, 1st edn. Springer Science + Business Media, Berlin, pp 211-239

Souza GM, Prado CHBA, Ribeiro RV et al (2016a) Toward a systemic plant physiology. Theor Exp Plant Physiol 28:341-346. https://doi.org/10.1007/s40626-016-0071-9

Souza GM, Bertolli SC, Lüttge U (2016b) Hierarchy and information in a system approach to plant biology: explaining the irreducibility in plant ecophysiology. In: Lüttge $U$ (ed) Progress in botany. Springer Science+Business Media, Berlin, pp 167-186

Takagi D, Ishizaki K, Hanawa H et al (2017) Diversity of strategies for escaping reactive oxygen species production within photosystem I among land plants: P700 oxidation system is prerequisite for alleviating photoinhibition in photosystem I. Physiol Plant 161:56-74. https://doi.org/10. $1111 /$ ppl.12562

Vitolo HF, Souza GM, Silveira J (2012) Cross-scale multivariate analysis of physiological responses to high temperature in two tropical crops with C3 and C4 metabolism. Environ Exp Bot 80:54-62

Wegner LH, Luttge U (eds) (2019) Emergence and modularity in life sciences. Springer International Publishing, Berlin

Wegner LH (2019) Modularity versus emergence: how to cope with complexity in whole-plant physiology? In: Wegner L, Lüttge U (eds) Emergence and modularity in life sciences. Springer, Cham

Ziotti ABS, Silva BP, Sershen, Lima Neto MC (2019) Photorespiration is crucial for salinity acclimation in castor bean. Environ Exp Bot. https://doi.org/10.1016/j. envexpbot.2019.103845

Publisher's Note Springer Nature remains neutral with regard to jurisdictional claims in published maps and institutional affiliations. 